\title{
Application of Sepsis-3 Criteria to Korean Patients with Critical IIInesses
}

\author{
Jae Yeol Kim ${ }^{1 *}$, Hwan II Kim²*, Gee Young Suh ${ }^{3}$, Sang Won Yoon ${ }^{1}$, Tae-Yop Kim ${ }^{4}$, Sang Haak Lee ${ }^{5}$, \\ Jae Young Moon ${ }^{6}$, Jae-Young Kwon ${ }^{7}$, Sungwon $\mathrm{Na}^{8}$, Ho Geol Ryu ${ }^{9}$, Jisook Park ${ }^{10}$, Younsuck Koh ${ }^{11}$
}

${ }^{1}$ Department of Internal Medicine, Chung-Ang University College of Medicine, Seoul; ${ }^{2}$ Division of Pulmonary and Critical Care Medicine, Department of Internal Medicine, Hallym University Medical Center, Anyang: ${ }^{3}$ Department of Critical Care Medicine, Samsung Medical Center, Sungkyunkwan University School of Medicine, Seoul;: ${ }^{4}$ Department of Anesthesiology, Konkuk University Medical Center, Konkuk University School of Medicine, Seoul; ${ }^{5}$ Department of Internal Medicine, St. Paul's Hospital, College of Medicine, The Catholic University of Korea, Seoul; ${ }^{6}$ Division of Pulmonary and Critical Care Medicine, Department of Internal Medicine, Chungnam National University Hospital, Daejeon; ${ }^{7}$ Department of Anesthesia and Pain Medicine, Pusan National University School of Medicine, Busan; ${ }^{8}$ Department of Anesthesia and Pain Medicine, Yonsei University College of Medicine, Seoul; ${ }^{9}$ Department of Anesthesiology and Pain Medicine, Seoul National University College of Medicine, Seoul:; Department of Software Convergence, Seoul Women's University, Seoul; "11 Department of Pulmonary and Critical Care Medicine, Asan Medical Center, University of Ulsan College of Medicine, Seoul, Korea

Background: The 2016 Society of Critical Care Medicine (SCCM)/European Society of Intensive Care Medicine (ESICM) task force for Sepsis-3 devised new definitions for sepsis, sepsis with organ dysfunction and septic shock. Although Sepsis-3 was data-driven, evidence-based approach, East Asian descents comprised minor portions of the project population.

Methods: We selected Korean participants from the fever and antipyretics in critically ill patients evaluation (FACE) study, a joint study between Korea and Japan. We calculated the concordance rates for sepsis diagnosis between Sepsis-2 and Sepsis-3 criteria and evaluated mortality rates of sepsis, sepsis with organ dysfunction, and septic shock by Sepsis-3 criteria using the selected data.

Results: Korean participants of the FACE study were 913 (383 with sepsis and 530 without sepsis by Sepsis-2 criteria). The concordance rate for sepsis diagnosis between Sepsis-2 and Sepsis-3 criteria was 55.4\%. The intensive care unit (ICU) and 28-day mortality rates of sepsis, sepsis with organ dysfunction, and septic shock patients according to Sepsis-3 criteria were $26.2 \%$ and $31.0 \%, 27.5 \%$ and $32.5 \%$, and $40.8 \%$ and $43.4 \%$, respectively. The quick Sequential Organ Failure Assessment (qSOFA) was inferior not only to SOFA but also to systemic inflammatory response syndrome (SIRS) for predicting ICU and 28-day mortality.

Conclusions: The concordance rates for sepsis diagnosis between Sepsis-2 and Sepsis-3 criteria were low. Mortality rate for septic shock in Koreans was consistent with estimates made by the 2016 SCCM/ESICM task force. SOFA and SIRS were better than qSOFA for predicting ICU and 28-day mortality in Korean ICU patients.

Key Words: intensive care units; organ dysfunction; Sepsis; Sepsis-3; septic shock

\section{INTRODUCTION}

The definitions of systemic inflammatory response syndrome (SIRS), sepsis, severe sepsis, and septic shock were formulated by consensus during a meeting of the American College of Chest Physicians (ACCP) and the Society of Critical Care Medicine (SCCM) in 1991 [1]. Sepsis

\section{Original Article}

Received: October 18, 2018 Revised: December 11, 2018 Accepted: December 14, 2018

\section{Corresponding author}

Younsuck Koh

Department of Pulmonary and Critical Care Medicine, Asan Medical Center, University of Ulsan College of Medicine, 88 Olympic-ro 43-gil, Songpa-gu, Seoul 05505, Korea Tel: +82-2-3010-3134

Fax: +82-2-3010-6968 E-mail:yskoh@amc.seoul.kr

*The first two authors contributed equally to this study.

Copyright () 2019 The Korean Society of Critical Care Medicine

This is an Open Access article distributed under the terms of Creative Attributions Non-Commercial License (http:// creativecommons.org/li-censes/by-nc/4.0/) which permits unrestricted noncommercial use, distribution, and reproduction in any medium, provided the original work is properly cited. 
was defined as SIRS due to infection, presumed or confirmed. When sepsis was associated with organ dysfunction, hypoperfusion, or hypotension, it was defined as severe sepsis. Septic shock was designated as sepsis-induced hypotension persisting despite adequate fluid resuscitation.

These definitions were revised in 2001 during the International Sepsis Definitions Conference [2]. The 2001 task force expanded the lists of diagnostic criteria for SIRS and organ dysfunction. However, they did not revise for the definition of sepsis due to a lack of supporting evidence. Practically, the definitions of sepsis, septic shock, and organ dysfunction have remained unchanged for more than two decades.

Given these circumstances, a task force was appointed by the European Society of Intensive Care Medicine (ESICM) and the SCCM to prepare new definitions of sepsis. The clinical criteria for sepsis were formulated by a data-driven approach. The task force performed systematic review and metaanalysis of sepsis studies, a Delphi study, and cohort studies using the Surviving Sepsis Campaign registry [3]. The 2016 SCCM/ESICM task force introduced a new bedside clinical score, quick Sequential Organ Failure Assessment (qSOFA), which consists of three parameters (respiratory rate $\geq 22 / \mathrm{min}$, altered mentation, and systolic blood pressure $[\mathrm{BP}] \leq 100$ $\mathrm{mmHg}$ ). Patients with at least two of these three clinical variables are more likely to experience poor outcomes typical of sepsis [4]. The term "severe sepsis" was considered to be superfluous and has been discarded. Instead, the Sequential (sepsis-related) Organ Failure Assessment (SOFA) was chosen to measure organ dysfunction due to reliability and simplicity [5]. Organ dysfunction is represented by increase in SOFA score of 2 points or more. Septic shock was defined as vasopressor requirement to maintain mean arterial pressure $\geq 65$ $\mathrm{mmHg}$ and serum lactate level $>2 \mathrm{mmol} / \mathrm{L}(18 \mathrm{mg} / \mathrm{dl}$ ) in the absence of hypovolemia.

The efforts of the task force that derived and validated the Sepsis-3 definitions should be appreciated. Nevertheless, there are concerns regarding the Sepsis-3 definitions. Most problematically, the health data utilized for the Sepsis-3 were almost exclusively derived from the United States and Germany. The primary cohort at the University of Pittsburgh Medical Center (UPMC) with suspected infection included 148,907 encounters. European descents comprised most of the cohort (76\%), with African-Americans making up the second largest group (14\%). East Asian descents make up one of remaining $10 \%$ population. Therefore, generalizability of Sepsis-3 definitions to East Asian descents has not been validated.

In 2012, the Korean Society of Critical Care Medicine (KSC-

\section{KEY MESSAGES}

- The concordance rates for sepsis between Sepsis-2 and Sepsis-3 criteria were low, which might be associated with the weak performance of quick Sequential Organ Failure Assessment (qSOFA).

- The mortality rate of Korean patients with septic shock was consistent with estimates by the 2016 Society of Critical Care Medicine/European Society of Intensive Care Medicine task force.

- Not only SOFA but also systemic inflammatory response syndrome (SIRS) was better than qSOFA in predicting intensive care unit (ICU) and 28-day mortality in Korean ICU patients.

$\mathrm{CM}$ ) and the Japanese Society of Intensive Care Medicine (JSICM) published a joint study, the fever and antipyretics in critically ill patients evaluation (FACE) study which evaluated the relationships between fever, antipyretic treatment, and mortality in patients admitted to the intensive care unit (ICU) [6]. In the FACE study, patients were classified into two groups depending on the presence of sepsis at the time of ICU admission according to Sepsis-2 criteria. We hypothesized that it would be possible to evaluate the validity of Sepsis-3 criteria and the differential power of Sepsis-3 criteria in Koreans using of FACE study data.

\section{MATERIALS AND METHODS}

\section{The FACE Study}

The FACE study was a binational, multicenter, prospective, and observational study to assess the relationship between fever, antipyretic treatments, and mortality in patients admitted to the ICU. It was conducted at 25 hospitals, 10 in Korea and 15 in Japan. Adult patients who were expected to require intensive care for more than 48 hours were enrolled from September 1, 2009 to November 30, 2009. Patients were classified into two groups at the time of ICU admission, those with and without sepsis according to Sepsis- 2 criteria as judged by attending physicians. Of a total of 1,425 consecutive patients (606 with sepsis and 819 without sepsis), we identified 913 Korean patients to evaluate the utility of Sepsis-3 criteria. JSICM, although very interested in this project, did not participate because of difficulties in obtaining new Institutional Review Board (IRB) approval for using FACE data to evaluate Sepsis-3. 


\section{Measurement of Sequential (Sepsis-Related) Organ Failure Assessment (SOFA) Score}

In the FACE study, severity of disease was evaluated according to the Acute Physiology and Chronic Health Evaluation (APACHE) II score. APACHE II score does not assess bilirubin level or platelet count, which are essential for the measurement of hepatic and hematologic function in SOFA. Therefore, we were required to obtain new IRB permission to collect these two parameters at each hospital. Nine out of 10 Korean hospitals that participated in the FACE study agreed to proceed after IRB approval for the collection of data regarding bilirubin level and platelet count and the use of the FACE data set to evaluate the utility of Sepsis-3 criteria. This study was approved by IRB of Chung-Ang University Hospital (IRB No. 1612-008-16023).

\section{Demographics, Clinical Characteristics and Outcomes of Korean Patients Enrolled in the FACE Study}

Clinical characteristics (route of ICU admission, main reasons for ICU care, and comorbidity), therapeutic modalities (use of special clinical treatments such as steroids, vasopressor, mechanical ventilation, and extracorporeal membrane oxygenation) and clinical outcomes (ICU and 28-day mortality rate) were reanalyzed for Korean participants of the FACE study.

\section{Application of qSOFA, SOFA, and Septic Shock Criteria to FACE Study Participants}

Patients enrolled in the FACE study were divided into two groups, those with and without sepsis according to Sepsis-2 criteria (more than two SIRS parameters with presumed or confirmed infection). We evaluated the concordance rates for the diagnosis of sepsis between old and new criteria. Each component of the SOFA score (pulmonary, coagulation, hepatic, circulatory, neurologic, and renal A \& B) was calculated in patients with and without sepsis. Serum lactate level was combined with "persisting hypotension requiring vasopressors to maintain mean arterial pressure $\geq 65 \mathrm{mmHg}$ " to identify patients whose parameters were compatible with the new Sepsis-3 definition of septic shock.

Mortality Rates of Sepsis, Sepsis with Organ Dysfunction, and Septic Shock

Using FACE data, we evaluated the ICU and 28-day mortality rates for sepsis, sepsis with organ dysfunction, and septic shock by Sepsis-3 criteria. We compared the mortality rates of Korean sepsis patients with those included in the UPMC data.

\section{Statistical Analysis}

Continuous variables were presented as mean and standard deviation. Categorical data were presented as frequencies and percentiles. Intergroup comparisons of demographic and safety variables were performed by t-test or Wilcoxon rank sum test. Intergroup comparisons of categorical data were performed using the chi-square test or Fisher exact test. We applied Bonferroni corrections and used them to determine whether multiple comparisons of groups were significant. The area under the receiver operating characteristic curve (AUROC) was used to evaluate the discriminatory capacity of ICU and 28-day mortality among qSOFA, SOFA, and SIRS. We considered AUROC to connote poor discrimination at 0.6 to 0.7 , adequate discrimination at 0.7 to 0.8 , and good discrimination at 0.8 to 0.9 .

\section{RESULTS}

Clinical Characteristics of Korean Participants of the FACE Study (with Sepsis Defined by Sepsis-2 Criteria)

Of a total of 913 patients, we identified 383 with and 530 without sepsis. Patients with sepsis were older and more likely to be male. More patients were enrolled from the medical ICU (MICU) than the surgical ICU (SICU). The main reason for MICU care was respiratory problems, while it was cardiovascular problems for SICU care. Over half of MICU patients had comorbidities, with malignancy being the most common. Patients admitted to the SICU had fewer comorbidities than those admitted to the MICU. The most common cause of the SICU admission was liver cirrhosis (Table 1).

Treatment Modalities and Clinical Outcomes of Patients (with Sepsis Defined by Sepsis-2 Criteria)

Patients with sepsis had received more medical therapies such as antipyretics, systemic corticosteroid and vasopressor than those without sepsis. A higher proportion of patients with sepsis required extra-corporeal membrane oxygenation therapy and stayed longer in the ICU compared to those without sepsis. Sepsis patients had higher ICU and 28-day mortality rates than those without sepsis. Patients with sepsis were less likely to be discharged from the hospital or transferred to another hospital than patients without sepsis (Table 2).

\section{Application of Sepsis-3 Criteria to Korean Participants of the FACE Study}

Of 383 patients with sepsis (according to Sepsis-2 criteria), 210 met at least two of three qSOFA criteria (systolic blood pres- 
Table 1. Clinical characteristics of Korean patients with critical illnesses by Sepsis-2 criteria

\begin{tabular}{|c|c|c|c|}
\hline Variable & Patient with sepsis & Patient without sepsis & P-value \\
\hline No. of patients & 383 & 530 & - \\
\hline Age (yr) & $66(53-74)$ & $61(48-71)$ & $<0.001$ \\
\hline Male sex & $243(63.4)$ & $300(56.6)$ & 0.038 \\
\hline $\begin{array}{l}\text { Route of ICU admission } \\
\text { Surgical ICU }(n=328) \\
\text { After surgery } \\
\text { After emergency surgery } \\
\text { Medical ICU }(n=582) \\
\text { General ward } \\
\text { Emergency room } \\
\text { Other hospital }\end{array}$ & $\begin{array}{c}16 \\
6(37.5) \\
10(62.5) \\
367 \\
154(42.0) \\
203(55.3) \\
10(2.7)\end{array}$ & $\begin{array}{c}312 \\
243(77.9) \\
69(22.1) \\
215 \\
97(45.1) \\
112(52.1) \\
6(2.8)\end{array}$ & $<0.001$ \\
\hline $\begin{array}{l}\text { Main reasons for ICU care } \\
\text { Sepsis }(n=25) \\
\text { Trauma }(n=7) \\
\text { Malignancy }(n=2) \\
\text { Postoperative care }(n=114) \\
\text { Gastrointestinal }(n=81) \\
\text { Renal or metabolic }(n=63) \\
\text { Respiratory }(n=363) \\
\text { Cardiovascular }(n=198) \\
\text { Central nervous }(n=23) \\
\text { Others }(n=32)\end{array}$ & $\begin{array}{c}25(6.5) \\
0 \\
1(0.3) \\
2(0.5) \\
23(6.0) \\
24(6.3) \\
252(66.0) \\
17(4.5) \\
17(4.5) \\
21(5.5)\end{array}$ & $\begin{array}{c}0 \\
7(1.3) \\
1(0.2) \\
112(21.1) \\
58(11.0) \\
39(7.4) \\
111(21.1) \\
181(34.4) \\
6(1.1) \\
11(2.1)\end{array}$ & $\begin{array}{r}<0.001 \\
0.024 \\
1.000 \\
<0.001 \\
0.009 \\
0.508 \\
<0.001 \\
<0.001 \\
0.002 \\
0.006\end{array}$ \\
\hline $\begin{array}{l}\text { Comorbidity } \\
\text { Malignancy } \\
\text { Liver cirrhosis } \\
\text { End stage renal disease } \\
\text { Immunocompromised } \\
\text { Others }\end{array}$ & $\begin{array}{l}193(50.4) \\
126(65.3) \\
29(15.0) \\
18(9.3) \\
39(20.2) \\
20(10.4)\end{array}$ & $\begin{array}{r}148(28.0) \\
56(37.8) \\
57(38.5) \\
24(16.2) \\
19(12.8) \\
17(11.5)\end{array}$ & $\begin{array}{r}<0.001 \\
<0.001 \\
<0.001 \\
0.055 \\
0.073 \\
0.741\end{array}$ \\
\hline Posttransplantation & $6(1.6)$ & $24(4.5)$ & 0.013 \\
\hline
\end{tabular}

Values are presented as median (range) or number (\%).

ICU: intensive care unit.

Table 2. Treatments methods and clinical outcomes of Korean patients with critical illnesses by Sepsis-2 criteria

\begin{tabular}{|c|c|c|c|}
\hline Variable & Patient with sepsis & Patient without sepsis & P-value \\
\hline No. of patients & 383 & 530 & - \\
\hline Use of antipyretics & $180(47.0)$ & $184(34.8)$ & $<0.001$ \\
\hline Use of steroid & $183(47.8)$ & 117 (22.2) & $<0.001$ \\
\hline Use of vasopressor & $176(46.6)$ & $196(37.8)$ & 0.009 \\
\hline Mechanical ventilation ${ }^{a}$ & $245(64.0)$ & $316(56.3)$ & 0.183 \\
\hline $\operatorname{ECMO}(n=123)$ & $72(18.8)$ & $51(9.6)$ & $<0.001$ \\
\hline Duration of ICU stay $(n=823)^{b}$ & $5(3-10)$ & $3(2-5)$ & $<0.001$ \\
\hline Readmission to ICU & $18(4.7)$ & $27(5.1)$ & 0.786 \\
\hline \multicolumn{4}{|l|}{ Outcome } \\
\hline 28-Day mortality & $102(26.7)$ & $63(12.1)$ & $<0.001$ \\
\hline ICU mortality & $85(22.2)$ & 49 (9.2) & $<0.001$ \\
\hline Survival in general ward & $96(25.1)$ & $139(26.6)$ & 0.612 \\
\hline Discharge from hospital or transfer to another hospital & $159(60.9)$ & 301 (82.7) & $<0.001$ \\
\hline
\end{tabular}

Values are presented as number (\%) or median (range).

ECMO: extracorporeal membrane oxygenation; ICU: intensive care unit.

${ }^{a}$ Mechanical ventilation within the first 24 hours during ICU stay; ${ }^{b}$ Patients with sepsis, $n=336$; Patients without sepsis, $n=487$. 
Table 3. Application of Sepsis-3 criteria to Korean patients with critical illnesses

\begin{tabular}{|c|c|c|c|}
\hline Variable & Patient with sepsis & Patient without sepsis & P-value \\
\hline No. of patients & 383 & 530 & - \\
\hline $\begin{array}{l}\text { qSOFA score } \\
\text { Systolic BP } \leq 100 \mathrm{mmHg} \\
\text { Altered mentality } \\
\text { Respiratory rate } \geq 22 / \mathrm{min} \\
\text { qSOFA } \geq 2\end{array}$ & $\begin{array}{l}184(48.7) \\
164(43.4) \\
254(67.2) \\
210(55.4)\end{array}$ & $\begin{array}{l}149(28.8) \\
202(61.0) \\
294(57.3) \\
257(49.1)\end{array}$ & $\begin{array}{r}<0.001 \\
<0.001 \\
0.003 \\
0.063\end{array}$ \\
\hline $\begin{array}{l}\text { SOFA score } \\
\text { Pulmonary } \\
\text { Coagulation } \\
\text { Hepatic } \\
\text { Circulatory } \\
\text { Neurologic } \\
\text { Renal A } \\
\text { Renal B } \\
\text { Total score }\end{array}$ & $\begin{array}{l}2(1-3) \\
1(0-2) \\
0(0-1) \\
1(0-4) \\
1(0-2) \\
1(0-2) \\
0 \\
9(5-11)\end{array}$ & $\begin{array}{c}2(1-3) \\
1(0-2) \\
0(0-2) \\
1(0-3) \\
0(0-2) \\
0(0-1) \\
0 \\
7(5-10)\end{array}$ & $\begin{array}{r}0.428 \\
0.385 \\
0.065 \\
0.009 \\
0.012 \\
<0.001 \\
0.250 \\
0.001\end{array}$ \\
\hline $\begin{array}{l}\text { Septic shock } \\
\text { Persisting hypotension requiring vasopressors }(n=372) \\
\text { Lactate }>2 \mathrm{mmol} / \mathrm{L}(\mathrm{n}=329) \\
\text { Both (total } n=771)\end{array}$ & $\begin{array}{r}176(46.6) \\
141(40.5) \\
98 / 348(28.1)\end{array}$ & $\begin{array}{r}196(37.8) \\
188(44.4) \\
85 / 423(20.1)\end{array}$ & $\begin{array}{l}0.009 \\
0.273 \\
0.009\end{array}$ \\
\hline
\end{tabular}

Values are presented as number (\%) or median (range).

qSOFA: quick Sequential Organ Failure Assessment; BP: blood pressure.

sure $\leq 100 \mathrm{mmHg}$, altered mentality, or respiratory rate $\geq 22$ / $\mathrm{min}$ ) at the time of ICU admission (55.4\%). The positive rate of each parameter was $76.2 \%$ for systolic BP $\leq 100 \mathrm{mmHg}, 63.3 \%$ for altered mentality, and $88.6 \%$ for respiratory rate $\geq 22 / \mathrm{min}$, respectively. Meanwhile, 257 of 530 patients (49.1\%) without sepsis (according to Sepsis-2 criteria) satisfied at least two of three qSOFA criteria. The difference between the two groups was not statistically significant $(\mathrm{P}=0.063)$.

All patients (both with and without sepsis) had SOFA scores higher than 2 points at the time of ICU admission. Of 348 sepsis patients whose lactate level was available, $98(28.2 \%)$ had lactate level $>2 \mathrm{mmol} / \mathrm{L}$ and they had hypotension which required vasopressor therapy to maintain mean arterial pressure $\geq 65 \mathrm{mmHg}$. Meanwhile, $20.1 \%$ of non-sepsis patients met new Sepsis-3 septic shock criteria. The difference between the two groups was statistically significant $(\mathrm{P}<0.01)$ (Table 3).

Mortality Rates of Sepsis, Sepsis with Organ Dysfunction, and Septic Shock Patients (Classified by Sepsis-3 Criteria) The overall ICU and 28-day mortalities of Korean patients enrolled for FACE study were $14.7 \%$ and $18.1 \%$, respectively. The ICU and 28-day mortality rates of sepsis, sepsis with organ dysfunction, and septic shock patients according to Sepsis-3 definitions were $26.2 \%$ and $31.0 \%, 27.5 \%$ and $32.5 \%$, and $40.8 \%$ and $43.4 \%$, respectively (Figure 1 ).

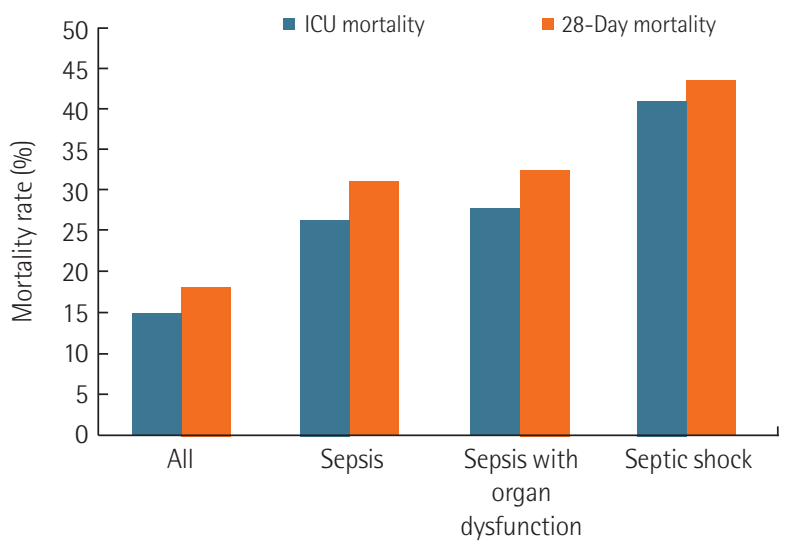

Figure 1. Mortality rates of all patients, patients with sepsis, patients with sepsis and organ dysfunction, and septic shock patients (classified by Sepsis-3 criteria) in Korean patients with critical illnesses who participated in the fever and antipyretics in critically ill patients evaluation study. ICU: intensive care unit.

\section{Discriminatory Capacity of qSOFA, SOFA, and SIRS for ICU and 28-Day Mortality in Sepsis Patients}

AUROCs for discriminatory capacity for ICU and 28-day mortality showed that qSOFA was inferior not only to SOFA but also to SIRS in predicting both parameters (Figure 2).

\section{DISCUSSION}

Sepsis is a clinical syndrome that comprises physiologic, bio- 

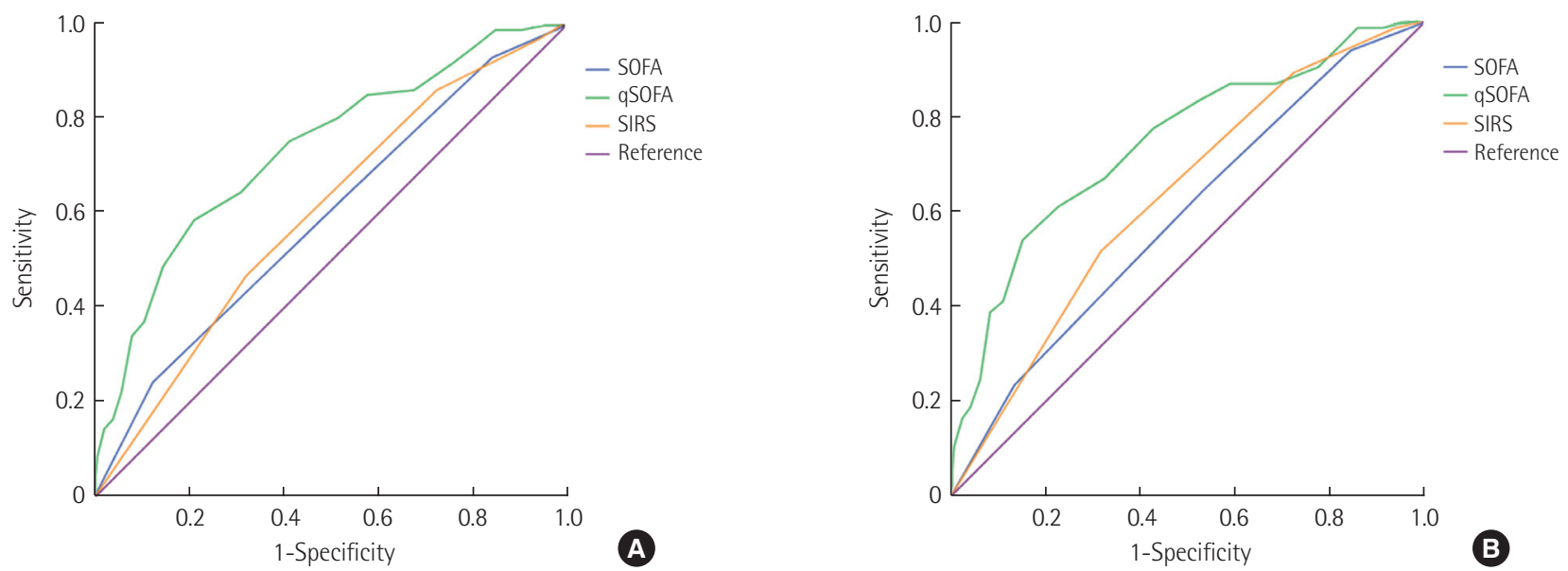

Figure 2. Area under the receiver operating characteristic curves (AUROCs) for discriminatory capacity for 28-day mortality and ICU mortality. (A) Twenty-eight-day mortality. AUROC: SOFA, 0.74 (95\% Cl, 0.68 to 0.79); qSOFA, 0.59 (95\% Cl, 0.53 to 0.66$)$; SIRS, 0.60 (95\% Cl, 0.54 to 0.66). (B) ICU mortality. AUROC: SOFA, 0.74 (95\% Cl, 0.68 to 081); qSOFA, 0.59 (95\% Cl, 0.52 to 0.66$) ;$ SIRS, 0.63 (95\% Cl, 0.57 to 0.70). SOFA: Sequential Organ Failure Assessment; qSOFA: quick SOFA; SIRS: systemic inflammatory response syndrome; Cl: confidence interval; ICU: intensive care unit.

logic, and biochemical abnormalities caused by inflammatory responses to invading microorganisms. The first international consensus conference in 1991 and subsequent revision in 2001 relied mainly on expert opinion to generate a clinical definition of sepsis $[2,7]$. Advances in the understanding of the pathophysiology of sepsis and the increasing availability of large electronic health record data sets, registries, and trial data sets enabled the application of an evidence-based approach to formulate new definitions of sepsis and septic shock. However, the lack of regional diversity among the patient sample upon which the Sepsis-3 criteria are based inspired us to evaluate the utility of these new sepsis definitions using data collected for the FACE study data.

The FACE study was a binational study performed in Korea and Japan, where most of population are East Asian descents. For the present study, we selected Korean participants of the FACE study. In the FACE study, patients were identified as with or without sepsis according to Sepsis-2 criteria at the time of ICU admission, which allowed us to figure out easily which patients harbored infection at the time of ICU admission [6].

The 2016 SCCM/ESICM task force for Sepsis-3 developed qSOFA, as a way to facilitate the identification of patients potentially at risk of dying from sepsis. The qSOFA score is easy to calculate since it has only three components (respiratory rate $\geq 22 / \mathrm{min}$, altered mentation, and systolic $\mathrm{BP} \leq 100$ $\mathrm{mmHg}$ ). A qSOFA score $\geq 2$ is associated with poor outcomes due to sepsis. The qSOFA score was originally recommended as for assessment of patients outside the ICU [3-5]. It has since been prospectively studied in several settings including the emergency department (ED) and ICU with conflicting results. In a 2018 meta-analysis of 38 studies that included patients from the ED, wards, and ICU, qSOFA showed poor sensitivity compared to SIRS for predicting mortality from sepsis [8]. In addition, a retrospective analysis of 184,875 ICU patients with infection-related admission reported that qSOFA was inferior to SOFA for predicting in-hospital mortality [9]. These findings are consistent with the results of the present study. We did not detect statistically significant differences between patients with or without sepsis by Sepsis- 2 criteria with qSOFA score $\geq 2$ criterion ( $55.4 \%$ vs. $49.1 \%$ ) (Table 3 ). qSOFA was originally developed as an assessment tool for patients outside the ICU. Because FACE study exclusively included patients who were expected to stay in the ICU for more than 48 hours, the weak performance of qSOFA in the present study is not surprising. In addition, the AUROC of discriminatory capacity for ICU and 28-day mortality showed that qSOFA score was inferior not only to SOFA and but also to SIRS in predicting both parameters (Figure 2). qSOFA may therefore not be as robust as originally anticipated. Among the three parameters comprising qSOFA, "altered mentality" showed the lowest positive rate in sepsis patients who met both Sepsis-2 and Sepsis-3 definition criteria. All things considered, clinicians should keep in mind that qSOFA was originally designed not as a diagnostic tool but rather as a predictive tool that calculates the risk of death from sepsis.

The term "severe sepsis," which describes sepsis with tissue hypoperfusion and/or organ dysfunction, is no longer in use 
in Sepsis-3 [2]. Instead, organ dysfunction is defined as an increase of two or more points in the SOFA score. Patients who fulfill these criteria have a predicted mortality of $10 \%$ or more. All patients enrolled in the FACE study satisfied the criterion of SOFA score greater than 2 points. The median values of SOFA in the with-sepsis and without-sepsis groups were 9 and 7 , respectively (Table 3). This finding implies that FACE patients are characterized by high degree of organ dysfunction, which might in turn explain the higher ICU and 28-day mortality rates of sepsis patients (26.2\% and $31.0 \%$ ) and sepsis with organ dysfunction (27.5\% and 32.5\%). Septic shock patients had ICU and 28-day mortality rates of $40.8 \%$ and $43.4 \%$, respectively (Figure 1). This is consistent with the estimates by the 2016 SCCM/ESICM task force. In their analysis, the mortality rate of septic shock patients was greater than $40 \%[10,11]$.

A limited number of studies evaluated the mortality rates of patients with sepsis using large samples of Korean patients. In a prospective, multicenter, observation study that included 1,192 adult patients admitted to 22 participating ICUs of 12 university hospitals in Korea, the 28-day mortality rate was $23.0 \%$ according to Sepsis-2 septic shock criteria [12]. The new Sepsis-3 definition of septic shock estimates $40 \%$ of mortality rate among septic shock patients, therefore, new definition may represent more severe disease than the previous definition. Another observational, prospective, multi-center registry study performed by 10 EDs at tertiary hospitals in Korea found that the 28-day mortality rate of septic shock patients defined according to Sepsis-3 criteria was $25.1 \%$, which is far lower than the mortality rate observed in the present study and that observed by the 2016 SCCM/ESICM task force [13]. To our best knowledge, this is the first study to present data regarding the mortality rates of patients with sepsis, sepsis with organ dysfunction, and septic shock as defined by Sepsis-3 criteria using a large sample of Korean ICU patients.

In conclusion, the concordance rates for sepsis between Sepsis-2 and Sepsis-3 criteria were low, which might be associated with the weak performance of qSOFA. The mortality rate of Korean patients with septic shock was consistent with estimates by the 2016 SCCM/ESICM task force. Not only SOFA but also SIRS was better than qSOFA in predicting ICU and 28-day mortality in Korean ICU patients.

\section{CONFLICT OF INTEREST}

No potential conflict of interest relevant to this article was reported.

\section{ORCID}

Jae Yeol Kim

https://orcid.org/0000-0003-3631-4610

Hwan Il Kim https://orcid.org/0000-0002-0165-5506

Gee Young Suh https://orcid.org/0000-0001-5473-1712

Sang Won Yoon

Tae-Yop Kim

Sang Haak Lee

Jae Young Moon

Jae-Young Kwon

Sungwon $\mathrm{Na}$

Ho Geol Ryu

Jisook Park

Younsuck Koh

https://orcid.org/0000-0003-0806-8969

https://orcid.org/0000-0001-6259-7656

https://orcid.org/0000-0001-8724-6289

https://orcid.org/0000-0002-7472-8278

https://orcid.org/0000-0002-1170-8042

https://orcid.org/0000-0001-8952-6049

https://orcid.org/0000-0002-9479-6710

https://orcid.org/0000-0001-5066-2027

\section{AUTHOR CONTRIBUTIONS}

Conceptualization: JYK, YK. Data curation: all. Formal analysis: HIK, SWY, JP. Funding acquisition: JYK, GYS, YK. Methodology: TYK, SHL, JYM, JYK, SN, HGR, JP. Project administration: JYK, YK. Visualization: JP, JYK. Writing - original draft: JYK, HIK, GYS, YK. Writing - review \& editing: all.

\section{REFERENCES}

1. American College of Chest Physicians/Society of Critical Care Medicine Consensus Conference: definitions for sepsis and organ failure and guidelines for the use of innovative therapies in sepsis. Crit Care Med 1992;20:864-74.

2. Levy MM, Fink MP, Marshall JC, Abraham E, Angus D, Cook D, et al. 2001 SCCM/ESICM/ACCP/ATS/SIS International Sepsis Definitions Conference. Crit Care Med 2003;31:1250-6.

3. Shankar-Hari M, Phillips GS, Levy ML, Seymour CW, Liu VX, Deutschman CS, et al. Developing a new definition and assessing new clinical criteria for septic shock: for the third International Consensus Definitions for Sepsis and Septic Shock (Sepsis-3). JAMA 2016;315:775-87.

4. Singer M, Deutschman CS, Seymour CW, Shankar-Hari M, Annane D, Bauer M, et al. The third International Consensus Definitions for Sepsis and Septic Shock (Sepsis-3). JAMA 2016;315:801-10.

5. Seymour CW, Liu VX, Iwashyna TJ, Brunkhorst FM, Rea TD, Scherag A, et al. Assessment of Clinical Criteria for Sepsis: for the third International Consensus Definitions for Sepsis and Septic Shock (Sepsis-3). JAMA 2016;315:762-74.

6. Lee BH, Inui D, Suh GY, Kim JY, Kwon JY, Park J, et al. Association of body temperature and antipyretic treatments with 
mortality of critically ill patients with and without sepsis: multi-centered prospective observational study. Crit Care 2012;16:R33.

7. Bone RC, Balk RA, Cerra FB, Dellinger RP, Fein AM, Knaus WA, et al. Definitions for sepsis and organ failure and guidelines for the use of innovative therapies in sepsis. The ACCP/ SCCM Consensus Conference Committee. American College of Chest Physicians/Society of Critical Care Medicine. Chest 1992;101:1644-55.

8. Fernando SM, Tran A, Taljaard M, Cheng W, Rochwerg B, Seely AJ, et al. Prognostic accuracy of the quick sequential organ failure assessment for mortality in patients with suspected infection: a systematic review and meta-analysis. Ann Intern Med 2018;168:266-75.

9. Raith EP, Udy AA, Bailey M, McGloughlin S, MacIsaac C, Bellomo R, et al. Prognostic accuracy of the SOFA score, SIRS criteria, and qSOFA score for in-hospital mortality among adults with suspected infection admitted to the intensive care unit.
JAMA 2017;317:290-300.

10. Casserly B, Phillips GS, Schorr C, Dellinger RP, Townsend SR, Osborn TM, et al. Lactate measurements in sepsis-induced tissue hypoperfusion: results from the Surviving Sepsis Campaign database. Crit Care Med 2015;43:567-73.

11. Cecconi M, De Backer D, Antonelli M, Beale R, Bakker J, Hofer C, et al. Consensus on circulatory shock and hemodynamic monitoring. Task force of the European Society of Intensive Care Medicine. Intensive Care Med 2014;40:1795-815.

12. Park DW, Chun BC, Kim JM, Sohn JW, Peck KR, Kim YS, et al. Epidemiological and clinical characteristics of communityacquired severe sepsis and septic shock: a prospective observational study in 12 university hospitals in Korea. J Korean Med Sci 2012;27:1308-14.

13. Ryoo SM, Kang GH, Shin TG, Hwang SY, Kim K, Jo YH, et al. Clinical outcome comparison of patients with septic shock defined by the new sepsis- 3 criteria and by previous criteria. J Thorac Dis 2018;10:845-53. 